\title{
Percepção da doença mental por profissionais de saúde: possibilidades de ampliação do cuidado
}

\section{Perception of mental illness by health professionals: possibility of broadening mental care}

\author{
Márcio Pinheiro Machado* \\ Secretaria Municipal de Botucatu, Botucatu, São Paulo, Brasil. \\ Maria Alice Ornellas Pereira \\ Universidade Estadual Paulista - UNESP, Botucatu, São Paulo, Brasil.
}

\begin{abstract}
RESUMO
O estudo abordou a percepção da doença mental por dezenove profissionais, membros da equipe de uma Unidade de Saúde de Família, através de representações gráficas (desenhos). Trata-se de pesquisa de natureza qualitativa. Para coleta de dados, foi utilizado o procedimento apresentativoexpressivo com tema. A partir da análise temática, emergiram quatro temas que apontaram para a doença mental ligada ao cuidado em saúde, à visão médico-centrada, à exclusão/inclusão e ao meio social. A análise identificou percepções associadas ao paradigma biologicista, com reprodução de preconceitos. Destacou-se a importância do cuidado ampliado, evidenciando dificuldades de atuação profissional. O estudo apontou para a necessidade de investimento na capacitação profissional, numa tentativa de transformação dos conteúdos ideoafetivos apresentados pelo imaginário dos sujeitos.

Palavras-chaves: Reabilitação Psicossocial, Desenhos, Pessoas mentalmente doentes, Equipe de assistência ao paciente, Saúde da família.
\end{abstract}

\begin{abstract}
This study approached perceptions of mental illness by nineteen professionals who work in a Family Health Unit, by means of graphic representations (drawings). We used qualitative methods. Data was collected by means of a Presentative-Expressive Procedure. Four themes were identified using Thematic Analysis. The professionals associate mental disorders with: health care, medical-centered view, exclusion/inclusion, social environment. These perceptions are related to a biological paradigm, favoring the reproduction of prejudices about mental illness. The analysis also emphasizes the importance of a wide range of cares, while considering how difficult it may be for the professionals. We conclude that it is necessary to invest on professional preparation, in an attempt to transform ideoaffective contents presented by the subjects' imaginary.

Keywords: Psychosocial rehabilitation, Drawings, Mentally Ill persons, Patient care team, Family health.
\end{abstract}




\section{Introdução}

O objetivo deste trabalho é estudar as percepções do transtorno mental, elaboradas por profissionais atuantes em equipe de saúde, por meio de pesquisa qualitativa, e a partir daí refletir sobre as possibilidades de se acolher e tratar adequadamente pessoas com sofrimento psíquico no âmbito da Atenção Primária à Saúde (APS).

A APS constitui uma forma de organizar os serviços de saúde, buscando integrar suas diversas dimensões com base nas necessidades da população. Em sua forma mais desenvolvida, a APS constitui-se na porta de entrada ao sistema e local responsável pelo cuidado à saúde dos indivíduos, famílias e da população, no decorrer do tempo (CONASS, 2007b). Segundo Giovanella (2008) o debate internacional e nacional sobre estratégias de APS foi incrementado na última década. Medidas para fortalecê-la foram incluídas nas reformas de saúde em diversos países europeus e latino-americanos. Conill (2008) revela que reformas em saúde realizadas em Québec, Canadá, promovem direitos em saúde numa conjuntura de controle de gastos, por meio da reorganização de novas práticas e estratégias para racionalizar a oferta, diminuir custos, facilitar o acesso e a coordenação dos cuidados.

No Brasil, a Estratégia Saúde a Família é responsável pela organização da APS no Sistema Único de Saúde (SUS), reafirmando uma prática integral, por meio da responsabilidade clínica e territorial (CONASS, 2007a). Nesse contexto, inclui-se ainda a atenção à saúde mental que, segundo o Ministério da Saúde, deve seguir um modelo de redes de cuidado, de base territorial e atuação transversal com outras políticas específicas, visando buscar o estabelecimento de vínculos e acolhimento. Baroni e Fontana (2009) entendem que a inclusão das ações de saúde mental na APS estende a atenção além das demandas mais graves de sofrimento psíquico, como as encontradas nos Centro de Atenção Psicossocial, ambulatórios especializados, hospitais-dias, entre outros, e se incorpora na porta de entrada do sistema de saúde, retornando ao lugar de origem, ou seja, a comunidade, a família, as relações cotidianas dos sujeitos.

$\mathrm{Na}$ perspectiva de outros autores (BÜCHELE; BORGES; LAURINDO; COELHO, 2006) a doença mental é, talvez, a que mais necessite da "solidariedade humana, do desprendimento, do destemor, de capacidades de absorção, de produção de sentimentos de cooperação e de integração social". Considerando a importância da dinâmica dos atores sociais na reconstrução e reconfiguração de teorias, Pereira (2007) aponta que os profissionais de saúde tem grande potencial para transformar, criar e edificar estratégias operativas no rumo da desinstitucionalização, contribuindo para a desconstrução de atitudes arraigadas. Esses esforços se integram no modelo da Reabilitação 
Psicossocial (SARACENO, 1998; 2001), que se baseia nos conceitos de autonomia, socialização, cidadania e contratualidade.

\section{Método}

A presente investigação é de natureza qualitativa, utilizando a técnica de desenho-história para estudar a percepção da doença mental por profissionais membros da equipe de uma Unidade de Saúde de Família (USF).

Os sujeitos da pesquisa pertencem à equipe de saúde envolvida na assistência numa USF do interior paulista, que se encontra integrada na rede de serviços municipais, e abriga duas equipes de saúde. Cada uma destas se constitui de médico, enfermeiro, auxiliares de enfermagem, agentes comunitários de saúde, dentista, auxiliar de dentista, auxiliar administrativo e auxiliar de serviços gerais, compondo um total de 19 sujeitos. O critério de escolha da unidade foi o tamanho da população atendida e a incidência de transtornos mentais. Como critério de seleção dos participantes, estabeleceu-se que fossem atuantes na USF, independentemente da categoria profissional e do tempo de trabalho.

A coleta de dados foi realizada após a obtenção de parecer favorável do Comitê de Ética em Pesquisa da Faculdade de Medicina de Botucatu-Unesp (Proc. 518/2007) dispondo da anuência dos sujeitos e seguindo a execução dos procedimentos éticos exigidos. Foram realizadas entrevistas individuais, em agosto de 2008, em sala reservada no interior da USF. Utilizou-se a técnica projetiva denominada Procedimento Apresentativo-Expressivo com Tema (PAET) desenvolvido por Vaisberg (2004), a partir da técnica de desenho-estória. Solicita-se ao entrevistado que elabore um desenho, dê um título para a sua produção e crie uma história relacionada ao tema abordado. Utilizamos folha de sulfite, lápis preto e lápis coloridos. Foi solicitado a cada participante a elaboração de um desenho, no qual pudesse expressar sua ideia acerca da doença mental. Em seguida, foi solicitada a atribuição de um título para o desenho produzido, assim como a criação de uma história pertinente ao desenho realizado.

A utilização desta técnica se justifica devido à possibilidade de expressão de vivências subjetivas de difícil verbalização. Segundo o referencial teórico utilizado (VAISBERG; CORREA; AMBRÓSIO, 2000), o ato de desenhar permite aos sujeitos entrarem numa área transicional, isto é, num espaço intermediário de experimentação entre seu mundo interior e exterior, facilitando a emergência de substratos inconscientes. A proposta da pesquisa qualitativa aplicada a profissionais da Estratégia Saúde da Família (ESF) coaduna com esta possibilidade ao analisar conteúdos ideoafetivos que emergiram 
durante a aplicação do PAET. A utilização deste procedimento de pesquisa veio contribuir para que os conteúdos não conscientes pudessem ser analisados, uma vez que a abordagem lúdica possibilita o deslocamento da atenção do sujeito para a área ideoafetiva e favorece a emersão de conteúdos associados ao tema da doença mental. Com o material obtido nas entrevistas, a partir da aplicação do PAET, foi utilizada a Atenção Flutuante' definhada por Laplanche e Pontalis (1995) como o ato da leitura repetitiva dos textos coletados. A seguir, foi utilizada a Análise Temática proposta por Bardin (1988) para a interpretação das histórias, possibilitando a busca de temas significativos a partir de um recorte transversal sobre os conteúdos registrados pelos sujeitos durante a entrevista.

\section{Apresentação e discussão dos resultados}

O material produzido se apresentou rico em significados. Foram apreendidos quatro temas, sugerindo que para os sujeitos a doença mental está associada: 1 ) ao cuidado em saúde; 2 ) à visão médicocentrada; 3) à exclusão/inclusão; 4) ao meio social. Cada um desses temas será discutido a seguir, apresentando um desenho-história para cada tema analisado.

\section{Doença mental ligada ao cuidado em saúde}

A partir das produções elaboradas foi possível constatar que os sujeitos atribuem ao cuidado um papel determinante, tendo ênfase no encontro, no acolhimento, no vínculo e na promoção da autonomia das pessoas assistidas. Na história criada a partir do desenho, 0 diálogo e a escuta do outro são vivenciadas pela pessoa, em sua relação ao profissional, como um cuidado esperado e resolutivo. Assim, configura-se à ideia, a emergência do vetor da solidariedade na relação terapêutica.

No desenho intitulado Depressão, contido na Figura 1, foi produzida a história que tem como personagem uma dona de casa que tendo muitos desejos frustrados ligados às possibilidades de autonomia, somados à baixa estima, contribuindo com o adoecimento. Porém, a depressão vivida pelo personagem, é narrada pelo sujeito como sendo uma fase difícil, mas que foi atenuada com o processo da escuta recebida. 


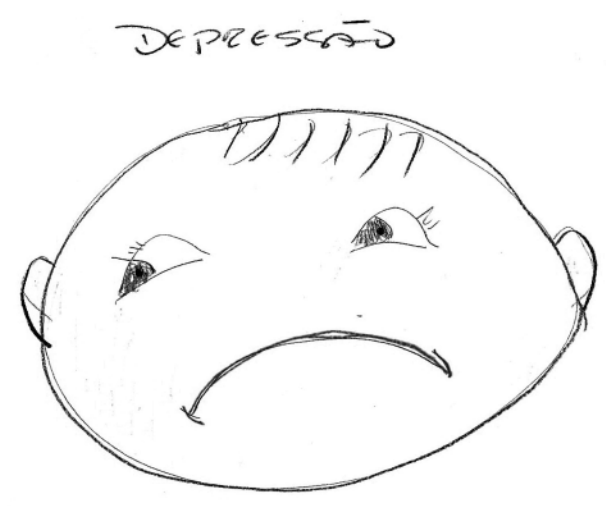

Figura 1 - Depressão.

[...] Foi só uma fase e hoje esta mulher está bem, feliz e nem precisou trabalhar, só com uma boa conversa e apoio de amigos e família melhorou (S03).

A narrativa sugere a importância do encontro intersubjetivo que favoreceu o processo de mudança do núcleo doença para a busca de possibilidades. Fica implícita a ideia de que o processo saúde/doença mental/cuidado está intrinsicamente ligado à busca de recursos internos e externos que sejam geradores de melhorias na dinâmica da subjetividade vivida. Saraceno (2001) evidencia a necessidade de se dar crédito às pessoas, a fim de que a autonomia encontre ocasião de expressão, o que pode favorecer que cada um seja empreendedor de si, prescindindo de uma rede social.

No desenho-história criado e posteriormente intitulado Expressões Difícieis, o sujeito desenha vários rostos com expressões humanas de dor, alegria, choro, espanto e fala da condição de ser continente ao outro. A história narra o encontro do sujeito com uma pessoa que apresentava-se chorosa, angustiada, desesperançada. A partir da escuta e do vínculo estabelecido, estando mais calma e sentindo-se apoiada, essa pessoa revela que estava precisando de muita atenção.

[...] com a expressão de satisfação por ter desabafado, a mesma me falou: - muito obrigado, uma estranha me ajudou muito mais, sem imaginar, do que muitos que convivem comigo há anos (S14).

O conteúdo produzido associa a doença mental ao estabelecimento da confiança, da escuta acolhedora, o que nos leva a refletir que não se trata de apenas uma conversa revestida de fantasia acolhedora, mas de um efetivo diálogo desenvolvido para auxiliar o outro na compreensão dos conflitos, que para tanto, necessita de tempo e disposição interna de quem é interlocutor. Ao sentir-se acolhida, a pessoa acometida pelo sofrimento psíquico, passa a apresentar-se 
verdadeiramente no encontro, o que poderá favorecer a organização interna dos conteúdos emocionais afetados. Desse modo, no encontro de pessoas pode haver o imaginário emocionalmente maduro, em que o outro é visto como um ser humano em sofrimento. Caso esse imaginário for emocionalmente imaturo, pode ser um forte impeditivo para que os profissionais de saúde consigam estabelecer um ambiente bom, e capaz de ser transformador da realidade do indivíduo. Assim, quando o imaginário dos profissionais de saúde não é suficientemente maduro, há dificuldade em preocupar-se com o outro e estabelecer determinantes para que a relação terapêutica se estabeleça, pois a sua realidade interna fica voltada essencialmente para si e gera dificuldade em preocupar-se com o outro que possui um sofrimento e necessita de cuidado (RIBEIRO; TACHIBANA; AIELLO-VAISBERG, 2008).

Vê-se que há mais questões subjacentes no encontro entre o profissional-paciente do que aquilo que efetivamente é considerado e instituído. Verifica-se que o cuidado pode ser afetado pela condição do profissional em estar diante do outro que traz a pluralidade e as singulares demandas.

\section{Doença mental ligada à visão médico-centrada}

Os conteúdos produzidos sugerem haver a ideia de doença mental ligada ao modelo médico. Contrariamente, o cuidado que tem o usuário como centro, pode promover a construção de vínculos e compromissos entre usuários e profissionais, a fim de prover necessidades individuais e coletivas.

No entanto, encontramos nas elaborações dos sujeitos a compreensão de doença mental ligada aos fatores biológicos. Algumas histórias descreveram a importância do atendimento centrado no médico e no tratamento medicamentoso. No desenho pertencente à Figura 2, que tem como título Igualdade e Diferenças, a história elaborada, evidencia a internação psiquiátrica como prática de tratamento, sem a apresentação da alternativa dos serviços substitutivos. 

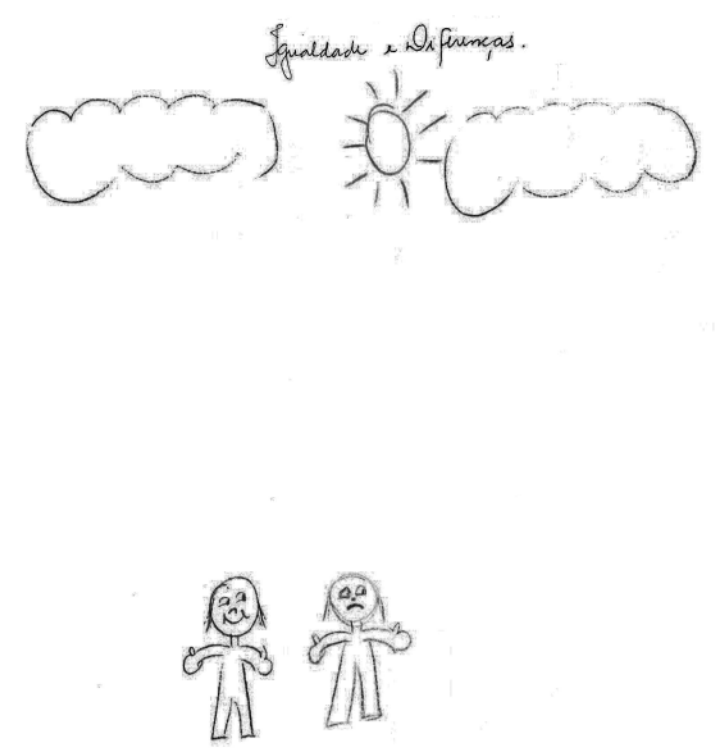

Figura 2 - Igualdade e Diferenças.

[...] A paciente Mara, chega à unidade de saúde, na qual faz acompanhamento de Saúde Mental há um ano. A paciente foi atendida por um profissional que na consulta falou para a paciente: Mara, você não tem como voltar a trabalhar, pois não está mais capacitada para suas funções. Após dois dias, a paciente estava internada no Hospital Psiquiátrico (S08).

Percebe-se no conteúdo uma atitude nova, o acompanhamento da pessoa com doença mental na unidade básica, concomitantemente com uma atitude da psiquiatria tradicional, a internação em hospital psiquiátrico como único recurso. Assim, chama a atenção na história, a atitude dos profissionais que não buscam assumir uma prática e condição antimanicomial de tratamento, reforçando práticas da psiquiatria tradicional que privilegiam a medicalização da doença e a centralidade na atuação do profissional médico.

O desenho-história intitulado Minha cura através da medicina mostra três rostos iguais, com diferentes expressões. No primeiro, a expressão é bastante carregada, tendo na lateral a imagem de um raio, no segundo, a expressão é menos fechada e, na lateral uma lâmpada, já o terceiro mostra o mesmo rosto, porém, com a expressão sorridente tendo ao lado uma estrela. Percebe-se que o sujeito narra a própria história, e que não é incomum que um profissional de saúde se encontre em situação de adoecimento, evidenciando que atitudes podem ser influenciadas e reforçadas pelos padrões culturais dominantes. Isso foi sugerido no título que apresenta a ideia de tratamento pautado pelo atendimento médico, além da atitude de delegar o cuidado por inteiro a este profissional, 0 que sustenta a dinâmica médico-centrada.

[...] Eu estava com depressão. Procurei ajuda e percebi de uma vez, que era pior do que eu pensava e não admitia. Eu 
estava com problema mental. Só um profissional da área poderia me ajudar (S17).

Os cenários de atendimento à saúde são percebidos conforme sua funcionalidade médica, enquanto que os fenômenos ou vivências necessitariam de análise mais integrativa, possibilitando múltiplas maneiras de atuação de uma equipe multiprofissional.

\section{Doença mental ligada à exclusão/ inclusão}

A noção dualista de exclusão/inclusão associada à doença mental emerge das produções elaboradas pelos sujeitos que relatam a discriminação e o preconceito que as pessoas com doenças mentais sofrem na sociedade. Paralelamente, aparece a ideia que 0 tratamento adequado pode possibilitar a inserção social. Por vezes, a ambivalência é percebida nas histórias que referem o atendimento em saúde mental prestado por profissionais, revelando quanto a problemática que envolve essa área é complexa e apresenta dificuldades a serem superadas.

Assim, os sujeitos do estudo se aproximam do presente tema, quando nas histórias, é destacada a necessidade de ir ao encontro do que é prioritário à pessoa adoecida, buscando a resposta dos dilemas que se impõem à saúde mental, conforme mostra o desenho e a história referente à Figura 3, que teve como título Exclusão Social.

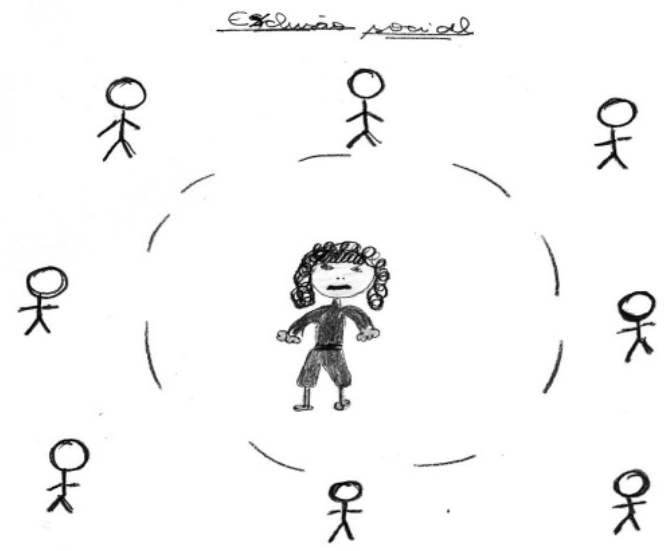

Figura 3 - Exclusão social.

[...] O indivíduo está presente na sociedade, mas ao mesmo tempo é diferenciado pelos demais cidadãos. Será que somente os que se consideram normais tem preconceitos, e por isso ocorre exclusão, ou são os próprios indivíduos que se excluem? (S02). 
Na elaboração apreende-se a noção de doença mental como dual e presente na sociedade, sendo reveladora de preconceitos cristalizados na conduta das pessoas que deles são alvo, como também na atitude dos demais. A produção de conceitos que pairam no meio social determina condutas que podem ser promotoras de inclusão ou determinantes para a exclusão. A psicodinâmica do preconceito vivido é capaz de gerar um sentimento de autoexclusão na pessoa acometida pela doença mental a partir da desconsideração que enfrenta diante da família e da sociedade, restando-Ihe a alternativa de acreditar na representação produzida. Essa representação pode conduzir a uma espiral de fracassos, onde somente a pessoa será responsabilizada por sua condição de adoecimento (SPIVAK, 1987).

Pode-se considerar que a inclusão/exclusão expressa a habilidade e desabilidade com que os profissionais do serviço de saúde promovem a tarefa de cuidar da pessoa que, em dado momento de sua vida, perdeu a capacidade de gerar sentido.

O profissional pode apresentar dificuldade de atuação quando o contato com pacientes internados em manicômio foi sua única experiência em saúde mental, conforme foi sugerido na produção do desenho de um grande pátio, um grande pavilhão intitulado Pacientes do Cantídio de Moura Campos, nome do tradicional hospital psiquiátrico existente no município pesquisado.

[...] quando fiz estágio lá de início tive medo, pois as pessoas falavam absurdo, mas depois vi que não era como pintavam. Alguns pareciam crianças grandes [...]. São pessoas alegres que só precisam de compreensão e ajuda. Ás vezes agressivas, mas isso ocorre por sua doença (S05).

A história remete ao medo inicial derivado da representação de doença mental que predomina na sociedade. Esse sentimento se transforma com a percepção do outro e provoca o desejo do profissional de incluir o doente mental à comunidade. No entanto, revela também desejo de tutela, pois paira a crença da impossibilidade da autonomia de decisão. Pensando na dinâmica da prática realizada pelos profissionais nos serviços, emerge a crítica que considera "mesmo quando atuante em serviços com intervenção biopsicossocial sobre a doença mental, tende a ser muito bio, um pouquinho psico e muito pouco social" (SARACENO, 1998, p.28). Pois nestes serviços são oferecidos um pouco de fármaco, um pouco de conversa terapêutica, sem mudança da situação de doença, que não é isolada no biológico do indivíduo, e que muitas vezes desconsidera um contexto mais amplo; sem a percepção de que os tratamentos são destinados a sujeitos e não aos seus diagnósticos (SARACENO, 1998). 


\title{
7 Doença mental ligada ao meio social
}

Os sujeitos trazem nas produções elaboradas as situações contextuais vividas, onde se verifica a íntima relação entre doença mental e meio social, tecendo assim conceitos acerca da doença mental que envolvem a influência do meio no desenvolvimento e permanência do estado de doença.

O meio social pode ser considerado como ferramenta de transformação para a mudança que se pretenda promover nas relações intrínsecas que a pessoa necessita para ser reabilitada. Consequentemente, saber atuar nas condições de sua governabilidade e expandi-la pode contribuir para que o profissional consiga realizar o que foi programado no projeto terapêutico do doente mental, como o indicado pelo desenho relativo à Figura 4, intitulado Resolutividade?

\section{Resoluturibce?}

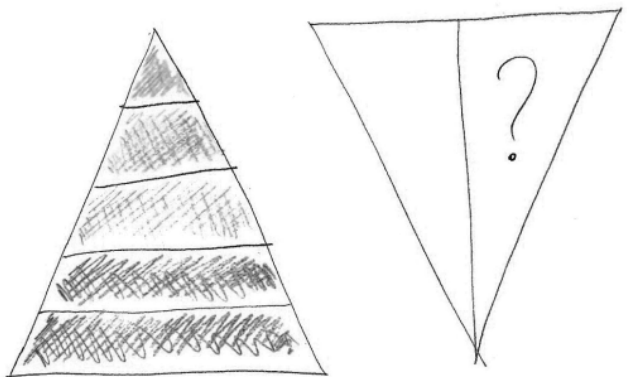

Figura 4 - Resolutividade?

\begin{abstract}
Assim, mesmo se encaminharmos para serviços mais especializados talvez também não iremos conseguir uma resolutividade tão alta, pois podemos até medicar ou tentar mudar o modo como as pessoas enxergam seus problemas/doenças, porém não podemos mudar o meio (S01).
\end{abstract}

O conteúdo da história produzida sugere que os serviços especializados não conseguirão sozinhos produzir o cuidado. Além disso, traz a ligação entre doença mental e o meio no qual o paciente vive e indica a corresponsabilidade pelo tratamento que deve existir entre a unidade de saúde e outros serviços.

Há que se destacar a micropolítica das relações sociais como espaço do encontro e produção da vida, o que permite a ressignificação das cenas experienciadas, que podem ter novos sentidos no mesmo local em que houve a interdição do indivíduo. "Então, não se trata de retirá-lo de seu ambiente, mas colaborar para que se mantenha ali, apesar da problemática instalada" (MERHY; FRANCO, 2003, p.319). 
Isso aciona os processos relacionais, cooperativos que visem a produção de vida e que ampliem as redes de dependências que compõem a sociedade.

A produção gráfica intitulada Indiferença contém três pessoas em um ambiente de lazer, sendo que uma delas está distanciada, sozinha e com expressão de tristeza e na história do desenho, a narrativa:

Quando uma pessoa tem algum problema mental, geralmente sente-se solitário, não encontra sentido para viver. Acha sempre que é um estorvo na vida de outras pessoas que vivem ao seu redor [...] (S11).

O conteúdo elaborado sugere que o sentimento de rejeição vivido pela pessoa em sofrimento psíquico, pode conduzir à solidão. A ausência do sentimento de pertença à comunidade que a circunda pode dificultar o desenvolvimento de sentido para a vida pela inexistência de teia de solidariedade e de afetividade das relações sociais significativas. A partir do processo de desenvolvimento humano é possível afirmar que:

A criança quando chega ao estágio de desenvolvimento em que consegue perceber a si próprio e mais duas pessoas, acaba por descobrir-se no interior de uma estrutura familiar, presente na maioria das culturas. E é nesse triângulo simples que apresenta as dificuldades e também, toda a riqueza da experiência humana (WINNICOTT, 1990, p. 57).

No interior da família, pode avançar para outros relacionamentos mais complexos. Portanto, sendo a família o primeiro núcleo social, cenário de possibilidades de desenvolvimento e de acontecimentos humanos, pode-se considerar a relevância desse núcleo no enfrentamento das dificuldades pertinentes à doença mental, assim como a importância da sensibilização do profissional para essa temática no processo do cuidado.

\section{Considerações finais}

O estudo sugere que o modelo da ESF possibilita o processo de reabilitação psicossocial da pessoa acometida pela doença mental, por suas práticas acontecerem na comunidade e por suas modalidades de cuidado, que se afinam com os pressupostos da reabilitação. Entretanto, a assistência prestada ainda traz em seu bojo conceitos que remetem à psiquiatria tradicional.

A análise possibilitou identificar que os profissionais apresentam dificuldade em promover o acolhimento adequado do doente mental, ora por não o reconhecerem dentro da temática do cuidado em saúde; ora por enfrentarem dificuldades subjetivas para lidar com a 
doença mental. A partir de uma perspectiva biopsicossocial, os conteúdos produzidos sugerem que os sujeitos conseguem realizar a escuta e o acolhimento do doente mental, vislumbram o ambiente como fonte de adoecimento e percebem a necessidade da sua inclusão em projetos e programas para a reabilitação da pessoa.

Concluímos que não basta aos profissionais deterem o saber técnico, sendo necessário que compreendam a influência do social, e desenvolvam a capacidade de edificar e de construir estratégias operativas que visem a melhoria da assistência em saúde mental. Vislumbramos práticas que aconteçam na comunidade, em equipamentos de atenção básica, contando com a lógica do apoio matricial. O modelo que se configura acolhe o embate entre a intersetorialidade no campo do fazer, assemelhada à transdisciplinaridade na construção de um saber novo. Vê-se que além do compromisso ético e político dos gestores de cada município em implementar a saúde mental na atenção básica, os resultados da presente pesquisa apontam na direção da promoção de futuras construções e intervenções nos campos teórico e prático da assistência em saúde mental na comunidade, com vistas à ampliação do cuidado prestado.

\section{Referências}

BARDIN, L. Análise de conteúdo. 1. ed. Lisboa: Edições 70, 1988.

BARONI, D. P. M.; Fontana, L. M. Ações em saúde mental na atenção primária no município de Florianópolis, Santa Catarina. Revista Mental, Barbacena, ano VII, n. 12, p. 15-37, jan. 2009.

BÜCHELE, F.; LAURINDO, D. L. P.; BORGES, V. F.; COELHO, E. B. S. A interface da saúde mental na atenção básica. Cogitare Enfermagem, Curitiba, v. 11, n. 3, p. 226-233, set. 2006.

CONILL, E. M. Ensaio histórico-conceitual sobre a atenção primária à saúde: desafios para a organização de serviços básicos e da estratégia saúde da família em centros urbanos no Brasil. Cadernos de Saúde Pública, Rio de Janeiro, n. 24, sup 1, p. S7-S27, 2008. CONSELHO NACIONAL DE SECRETÁRIOS DE SAÚDE (CONASS). A estratégia saúde da família. In: - Atenção primária e promoção à saúde. Brasília: CONASS, 2007a, p. 74-97.

. Os fundamentos da atenção primária e da promoção da saúde. In: Atenção primária e promoção à saúde. Brasília: CONASS, 2007b, p. 36-51.

GIOVANELLA, L. Desafios para a organização de serviços básicos e da estratégia saúde da família. Cadernos de Saúde Pública, Rio de Janeiro, n. 24, sup 1, p. S21-S23, 2008.

LAPLANCHE, J. ; PONTALIS, J. B. Vocabulário de Psicanálise. 4. ed. São Paulo: Martins Fontes, 1995. 
MERHY, E. E.; FRANCO, T. B. Por uma composição técnica do trabalho centrada nas tecnologias leves e no campo relacional. Saúde e Debate, Rio de Janeiro, v. 27, n. 65, p. 316-323, set./dez. 2003. PEREIRA, M. A. O. Psychosocial rehabilitation in mental health care: strategies under construction. Revista Latino-Americana de Enfermagem, Ribeirão Preto, v. 15, n. 4, p. 658-64, jul./ago. 2007. RIBEIRO, D. P. S. A.; TACHIBANA, M.; AIELLO-VAISBERG, T. M. J. A experiência emocional do estudante de psicologia frente à primeira entrevista clínica. Aletheia, Canoas, n. 28, p. 135-145, dez 2008.

SARACENO, B. A concepção de reabilitação psicossocial como referencial para as intervenções terapêuticas em saúde mental. Revista Terapia Ocupacional da Universidade de São Paulo, São Paulo, v. 9, n. 1, p. 26-31, jan./abr. 1998.

. Libertando identidades: da reabilitação psicossocial à cidadania possível. 1. ed. Rio de Janeiro: TeCorá, 2001.

SPIVAK, M. Introduzioni alla riabilitazioni sociale: teoria, tecnologia e metodi di intervento. Rivista Sperimentale di Freniatria, Reggio Emilia, v. 106, n. 3, p. 522-74, dec. 1987.

VAISBERG, T. M. J. A.; CORREA, Y.B., AMBRÓSIO, F.F. Encontros brincantes: o uso de procedimentos apresentativos na pesquisa e na clínica winnicottiana. In: $9^{\circ}$ ENCONTRO LATINO-AMERICANO SOBRE O PENSAMENTO DE D. W. WINNICOTT, 2000, Rio de Janeiro. Anais... Rio de Janeiro: Grupo de Estudos Psicanalíticos, 2000. p. 331-341.

VAISBERG, T. M. J. A. Ser e fazer: enquadres diferenciados na clínica winnicottiana. 1. ed. São Paulo: Ideias e Letras, 2004.

WINNICOTT, D.W. 0 ambiente e os processos de maturação: estudos sobre a teoria do desenvolvimento emocional. 3. ed. Porto Alegre: Artes Médicas, 1990.

\author{
Endereço para correspondência \\ Márcio Pinheiro Machado \\ Secretaria Municipal de Saúde, Avenida Santana, n० 323, Centro, CEP \\ 18603-700, Botucatu, São Paulo, SP, Brasil. \\ Endereço eletrônico institucional: saudemental@botucatu.sp.gov.br \\ Maria Alice Ornellas Pereira \\ Faculdade de Medicina de Botucatu (UNESP), Campus Universitário Rubião \\ Jr, s/no, 18603-970, Botucatu, São Paulo, SP, Brasil. \\ Endereço eletrônico: malice@fmb.unesp.br
}

Recebido em: 20/05/2011

Reformulado em: 09/01/2012

Aceito para publicação em: 18/01/2012

Acompanhamento do processo editorial: Deise Mancebo

Notas

* Psicólogo Mestre pela EERP/USP, Articulador de Saúde Mental da Secretaria Municipal de Saúde de Botucatu, São Paulo, Brasil. 
Márcio Pinheiro Machado, Maria Alice Ornellas Pereira Percepção da doença mental por profissionais de saúde: possibilidades de ampliação do cuidado

** Enfermeira, Professora Doutora Adjunta da Faculdade de Medicina de Botucatu - Departamento de Enfermagem - Universidade Estadual Paulista (UNESP), Botucatu, São Paulo, Brasil. 